\title{
Colonic Perforation and Transstomal Evisceration of Small Bowel
}

\author{
Jack Cecire $^{\mathrm{a}, \mathrm{b}}$, Amit Sarkar ${ }^{\mathrm{a}}$, William Ross ${ }^{\mathrm{a}}$, Andrew Sutherland ${ }^{\mathrm{a}}$
}

\begin{abstract}
The formation of an abdominal stoma is a commonly performed procedure in gastrointestinal surgery. Common complications include parastomal hernia, stomal necrosis, stomal prolapse, stenosis and secondary effects to surrounding skin. Transstomal evisceration is an extremely rare complication with only two previous reported cases. We present a unique case of transstomal evisceration of small bowel secondary to blunt force trauma. In addition, parastomal evisceration is also uncommon with only 15 reported cases. These conditions require emergent operative intervention due to the risk of ischemic injury to eviscerated contents.
\end{abstract}

Keywords: Parastomal; Transstomal; Evisceration; Colostomy; Ileostomy; Prolapse; Hernia

\section{Introduction}

The formation of an abdominal stoma is a commonly performed surgical procedure, with a variety of indications. Common complications from formation of stoma include prolapse, parastomal hernia and peristomal skin complications with the total incidence of all-cause complications estimated at approximately $25 \%$ [1]. Parastomal/transstomal eviscerations are extremely rare complications with only 17 cases reported in the literature. The majority of these cases report early evisceration, within 2 weeks postoperatively, with abdominal contents herniating through the fascial defect and protruding externally via mucocutaneous separation at the stoma site. The other reported cases relate to late occurrences of evisceration with the vast majority also reporting separation at the mucocutaneous junction. In this paper, we present the unique case of a colonic perforation proximal to the stomal opening, with transstomal evisceration of small bowel.

Manuscript submitted March 3, 2021, accepted March 10, 2021

Published online March 23, 2021

aDepartment of Surgery, Coffs Harbour Health Campus, Australia

${ }^{b}$ Corresponding Author: Jack Cecire, Department of Surgery, Coffs Harbour Health Campus, 345 Pacific Hwy, Coffs Harbour, NSW 2450, Australia. Email: j.cecire@gmail.com

\section{Case Report}

A 77-year-old male presented to the emergency department with evisceration of small bowel into his stoma bag (Fig. 1). The event was preceded by a blunt force trauma to the abdomen. He had multiple previous abdominal surgeries due to secondary effects of Crohn's disease, including an open right hemicolectomy for terminal ileal disease and subsequent loop colostomy for rectal stricture causing recurrent large bowel obstructions failing medical therapy. Due to stomal prolapse, this had been converted to an end colostomy. In addition, he had previously required a laparotomy for perforated duodenal ulcer. The patient's medical history was also significant for chronic obstructive pulmonary disease for which he was on long-term immunosuppressive therapy with systemic corticos-

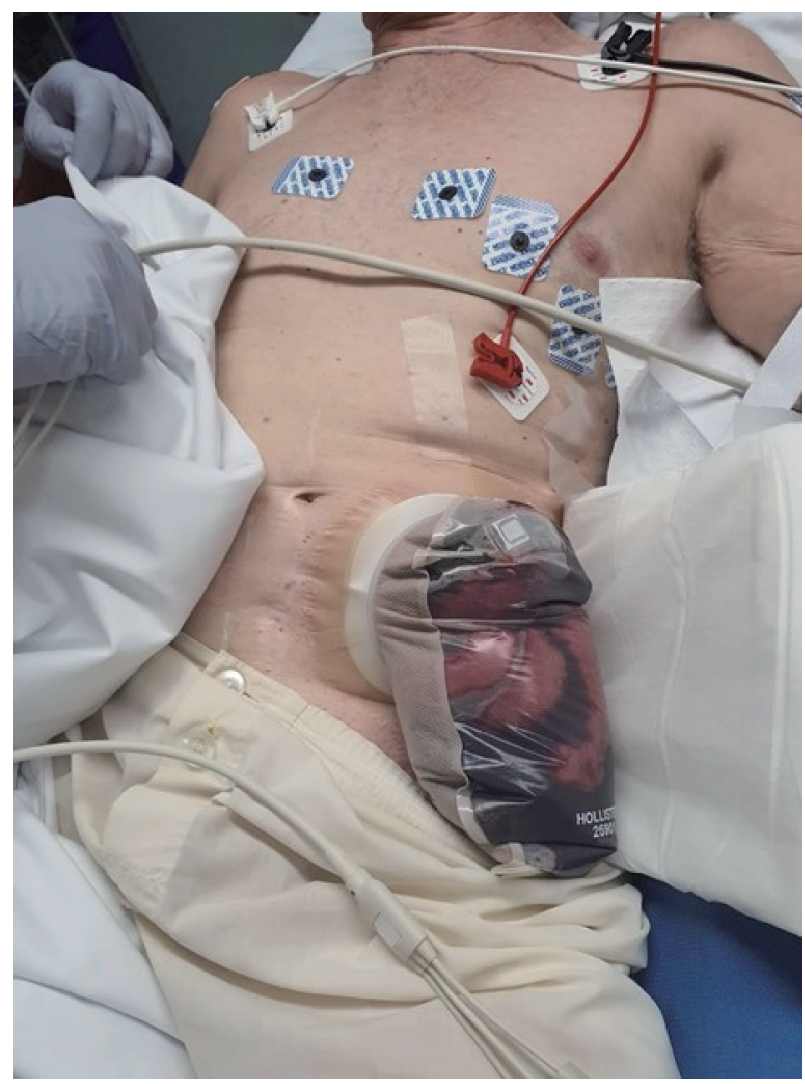

Figure 1. Eviscerated small bowel in stoma bag. 


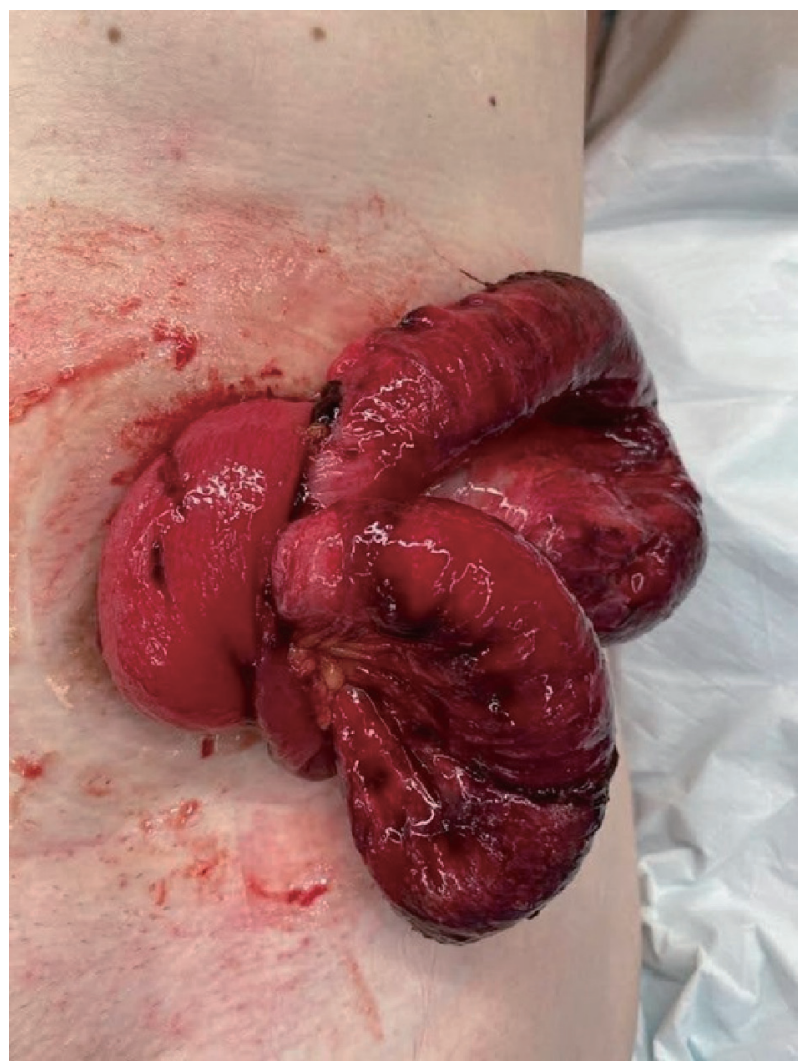

Figure 2. Eviscerated small bowel with signs of strangulation.

teroids. During a recent admission, the patient presented with small bowel obstruction secondary to adhesions. The episode had been managed nonoperatively and the obstruction settled completely. Within this admission the patient was noted to have a reducible stomal prolapse of $10 \mathrm{~cm}$ and a parastomal hernia containing small bowel.

At the time of the current presentation to the emergency department the patient was cleared of other injuries related to blunt abdominal trauma. The serosal surface of small bowel was noted in the stoma bag, which was left in place to protect the bowel. The patient was then taken to theater emergently as the eviscerated small bowel showed early signs of strangulation (Fig. 2). A laparotomy was performed and the eviscerated small bowel was reduced and deemed viable. The cause for his transstomal evisceration was identified as a large colonic perforation $10-15 \mathrm{~cm}$ proximal to the stoma, through which the small bowel protruded into the colonic lumen and then through the colostomy (Fig. 3). The perforation was in the region of colon that was known to prolapse at times.

There were no features in the colon to suggest ischemia or necrosis as a preceding contributor to the perforation. The operation was made complicated by the dense adhesions from multiple previous abdominal surgeries and his background of Crohn's disease. The stoma was resected up to the point of colonic perforation and refashioned through the same abdominal wall defect. There were two enterotomies, one of which was repaired primarily and the other requiring a small bowel resection. Postoperatively, his admission was complicated by

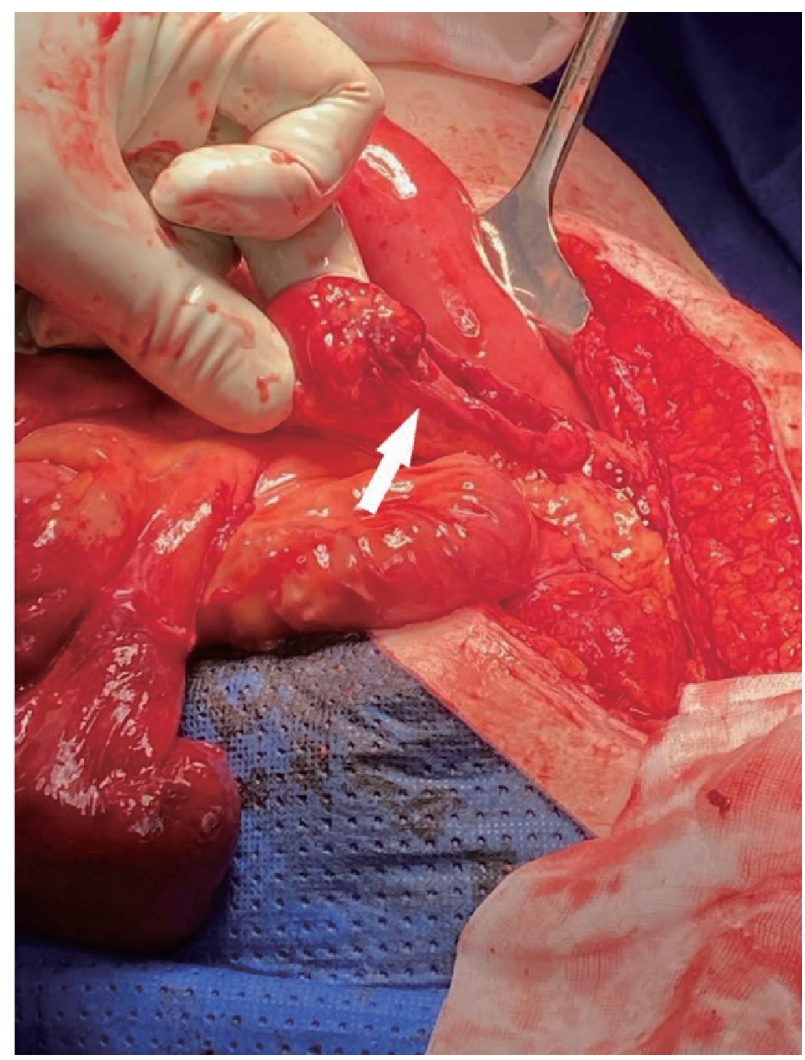

Figure 3. Site of colonic perforation.

a return to theater for management of a small bowel injury and nonoperative management of surgical site infection. After a short period of rehabilitation, the patient was discharged.

\section{Discussion}

Parastomal evisceration is an extremely rare complication post abdominal stoma formation with only 15 cases reported in the literature. There are even less cases of transstomal evisceration with only two cases identified on literature review. One of these cases of transstomal evisceration was secondary to a perforation resulting from ischemic colitis and the other identified vomiting as the precedent cause. This is the first reported case of transstomal evisceration arising from blunt force trauma.

Table 1 [2-17] summarizes the 17 cases of parastomal and transstomal evisceration. Of the 17 cases identified from the literature review, $14(82 \%)$ were male with a median age of 62 years. Twelve $(71 \%)$ of the stoma were colostomies and nine (53\%) were end stomas. Eight (47\%) of these were immediate or within 2 weeks of formation of the stoma. All of these acute cases involved dehiscence of the stoma site, with mechanical ventilation and severe bouts of coughing being identified as contributing factors.

There were eight reported cases of late parastomal/transstomal evisceration. Lapena-Rodriguez et al have previously identified stomal prolapse and parastomal hernia as possible factors present prior to evisceration, with these being present in all 

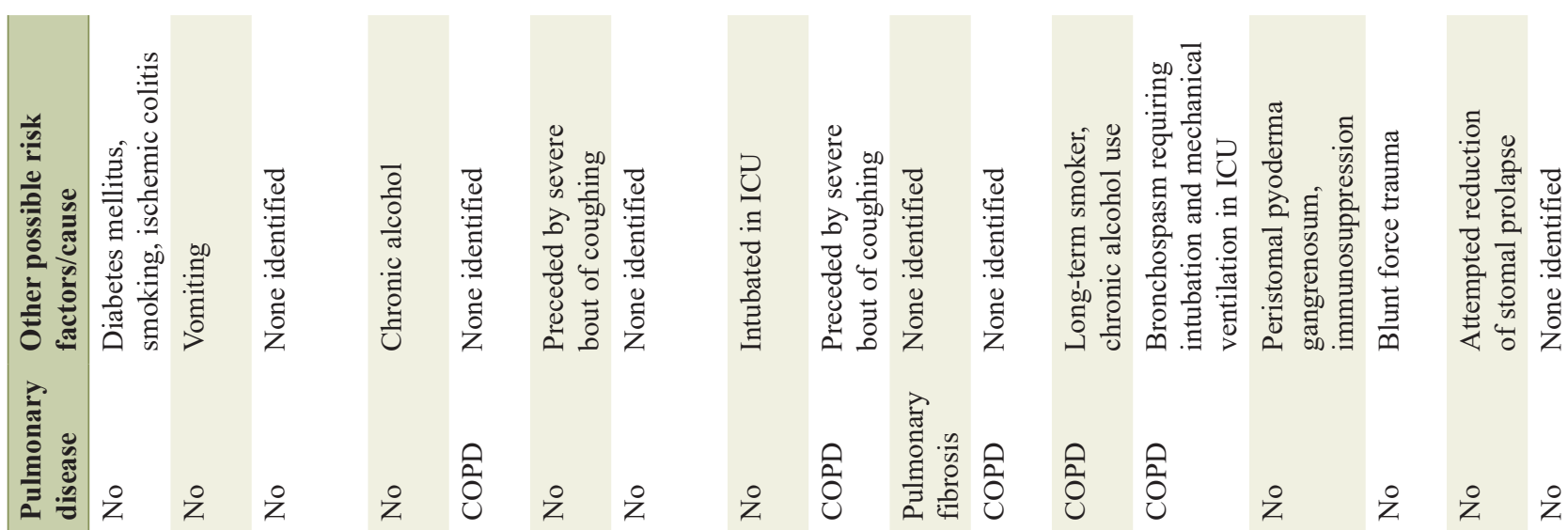

そ $\stackrel{2}{2}$ ลิ

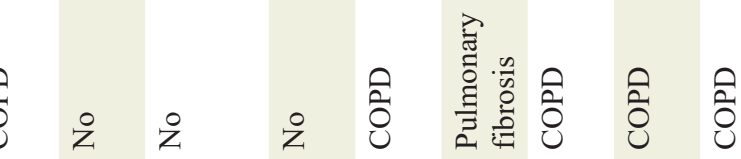

ప.

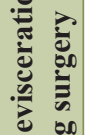

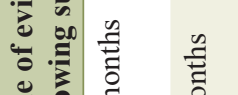

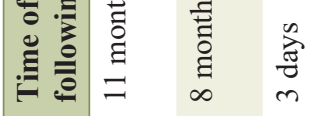

齐

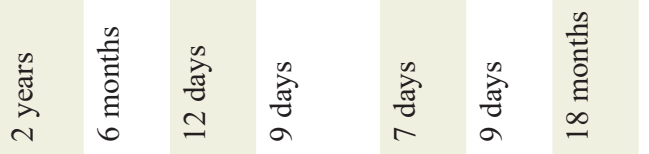

$\stackrel{\Xi}{\stackrel{0}{\pi}}$

疍
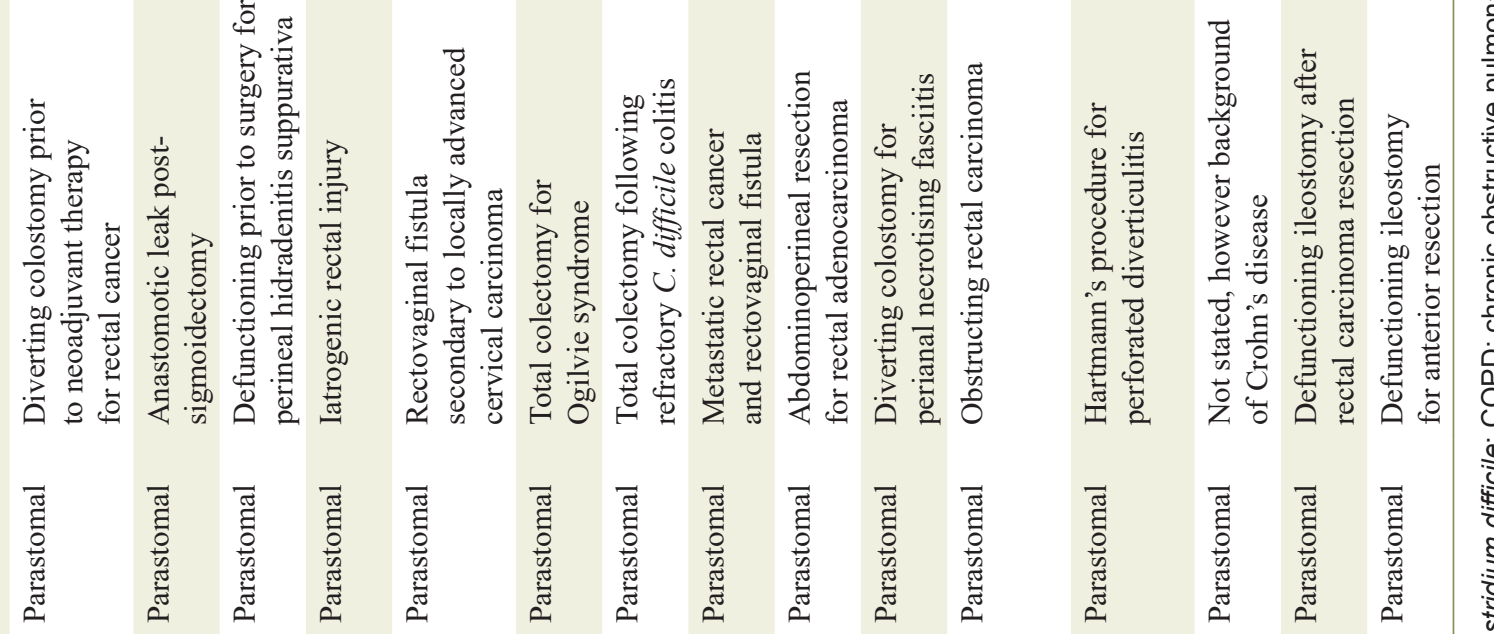

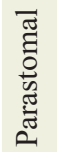

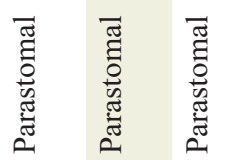

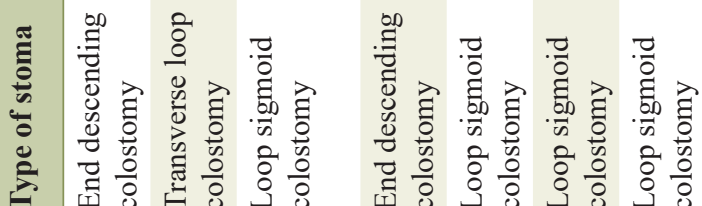
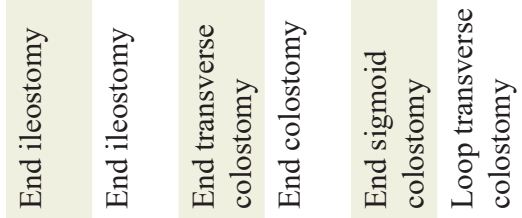

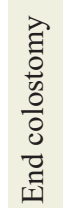
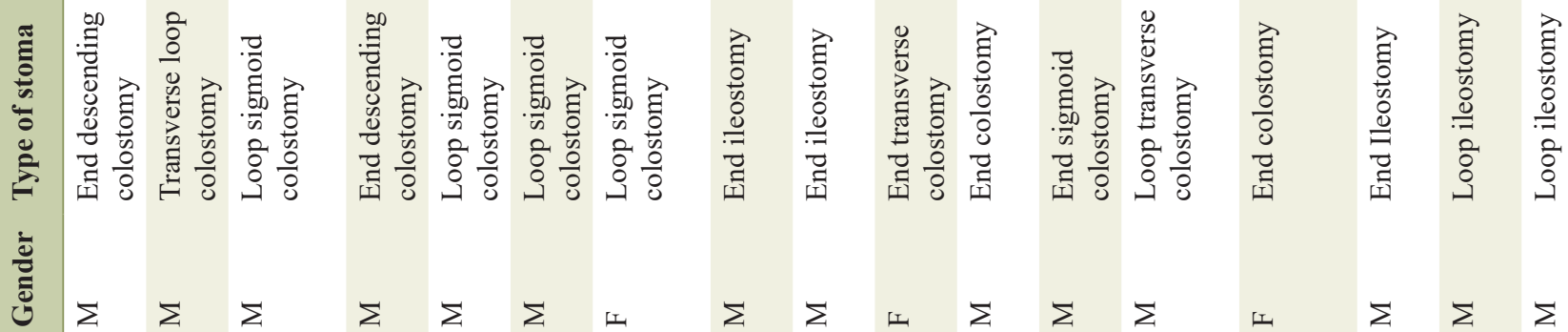

造

$\forall \quad \vec{n}$

$\stackrel{N}{\circlearrowright}$

$\frac{0}{\frac{\pi}{0}}$

$\frac{\circ}{\frac{0}{0}}$

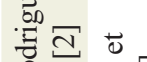

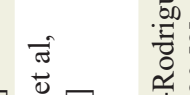

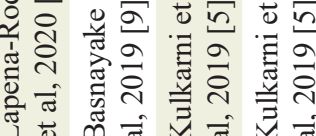

$\Sigma$

\&

$\infty \quad$

$\stackrel{+}{\infty}$

ช สู

๖

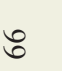

$\approx i \infty \quad n$

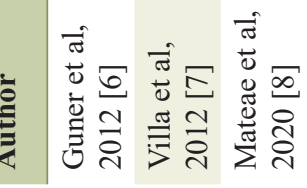

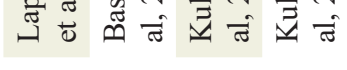

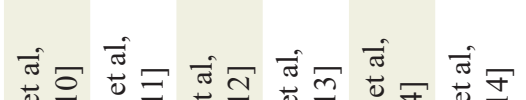

बं

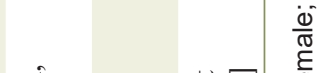

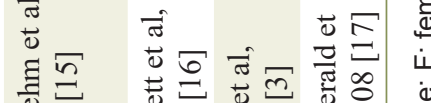

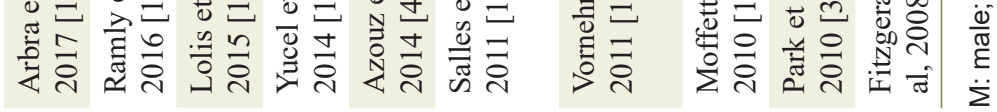


four cases of parastomal evisceration identified in their review [2]. Parastomal hernia, stomal prolapse, parastomal/transstomal evisceration all can result from increased intra-abdominal pressure [18]. In the cases of late evisceration identified in this study three out of eight (38\%) had underlying respiratory disease, either chronic obstructive pulmonary disease (COPD) or pulmonary fibrosis which probably contributes by the similar mechanisms including increased intra-abdominal pressures. One case resulted from attempted reduction of a stomal prolapse [3].

With stomal prolapse occurring in $2-3 \%$ of patients with a stoma and parastomal hernia in colostomies reported as high as $39 \%[1,19,20]$, it would be difficult to argue that these are sufficient risk factors to suggest stomal revision to prevent evisceration, given its extremely low incidence.

The principles of management in the cases identified in this review were for prompt operative intervention including reduction of the eviscerated abdominal contents, examining for viability, and performing resection if bowel is deemed nonviable and refashioning or resiting of the stoma.

The consequence of parastomal/transstomal evisceration can be severe including strangulation and subsequent necrosis of the eviscerated organ being the prominent concern. Resection of $100-\mathrm{cm}$ segment of small bowel was required in one case, and another reported a death related to fecal contamination, overwhelming sepsis and multi-organ failure $[4,5]$.

In conclusion, parastomal/transstomal evisceration is an extremely rare complication of stoma formation. Both early and late cases are related to raised intra-abdominal pressures, with early evisceration also resulting in stomal dehiscence. This case recognizes blunt force trauma as a further cause for presentation. Urgent operative intervention is required due to the risk of bowel ischemia.

\section{Acknowledgments}

None to declare.

\section{Financial Disclosure}

None to declare.

\section{Conflict of Interest}

None to declare.

\section{Informed Consent}

Informed consent was obtained prior to the completion of this case report

\section{Author Contributions}

J. Cecire and A. Sarkar contributed towards writing of the manuscript. W. Ross and A. Sutherland provided advice, review and alterations to the manuscript prior to submission.

\section{Data Availability}

The authors declare that data supporting the findings of this study are available within the article.

\section{References}

1. Malik T, Lee MJ, Harikrishnan AB. The incidence of stoma related morbidity - a systematic review of randomised controlled trials. Ann R Coll Surg Engl. 2018;100(7):501508.

2. Lapena-Rodriguez M, Fernandez-Moreno MC, MartinezMontava E, Munoz-Forner E, Ortega J. Late parastomal evisceration. Int J Colorectal Dis. 2020;35(9):1787-1789.

3. Park SJ, Lee SH, Lee KY. Small bowel evisceration by rupture of prolapsed loop ileostomy. Colorectal Dis. 2010;12(6):603-604.

4. Azouz V, Simmons JD, Abourjaily GS. Immediate postoperative parastomal end sigmoid hernia resulting in evisceration and strangulation. J Surg Case Rep. 2014;2014(5).

5. Kulkarni AA, Chauhan V, Sharma V, Singh H. Parastomal Evisceration: A Report of Two Cases and Review of Literature. Cureus. 2019;11(9):e5750.

6. Guner A, Kahraman I, Ozkan OF, Aktas A, Kece C. Transstomal small bowel evisceration after colonic perforation secondary to ischemic colitis. Case Rep Surg. 2012;2012:560683.

7. Villa M, Iannelli E, Grande M, Rossi P, Tucci G. An unusual case of small intestine evisceration through a transverse loop colostomy. Colorectal Dis. 2012;14(1):e27-28.

8. Mates IN, Gheorghe M, Tomsa R, Sumedrea EL. Paracolostomy evisceration: short review and a new case report. Chirurgia (Bucur). 2020;115(1):95-101.

9. Basnayake O, Jayarajah U, Jayasinghe J, Wijerathne PK, Samarasekera DN. Spontaneous rupture of a parastomal hernia with evisceration of small bowel: a case report. BMC Surg. 2019;19(1):43.

10. Arbra CA, Fann SA. Parastomal evisceration: rare complication after total abdominal colectomy. Am Surg. 2017;83(9):e379-e380.

11. Ramly EP, Crosslin T, Orkin B, Popowich D. Strangulated ileostomy evisceration following lateralizing mesh repair of parastomal hernia. Hernia. 2016;20(2):327-330.

12. Lolis ED, Savvidou P, Vardas K, Loutseti D, Koutsoumpas V. Parastomal evisceration as an extremely rare complication of a common procedure. Ann R Coll Surg Engl. 2015;97(7):e103-104.

13. Yucel AF, Pergel A, Aydin I, Sahin DA. A rare stoma-related complication: parastomal evisceration. Indian J Surg. 2014;76(2):154-155.

14. Salles VJA, Saba E, Pissinin ER, Arguello ERF, Machado Filho HN. Complication related to colostomy orifice: intestinal evisceration. Journal of Coloproctology (Rio de 
Janeiro). 2011;31(4):397-400.

15. Vornehm ND, Kelley SR, Ellis BJ. Parastomal small bowel evisceration as a result of parastomal pyoderma gangrenosum in a patient with Crohn's disease. The American Surgeon. 2011;77(7):150-151.

16. Moffett PM, Younggren BN. Parastomal intestinal evisceration. West J Emerg Med. 2010;11(2):214.

17. Fitzgerald JE, Tang SW, Lake EJ, Richards T, Acheson AG. Small bowel evisceration: a rare complication of laparoscopic ileostomy. Colorectal Dis. 2008;10(4):407-
408.

18. Hotouras A, Murphy J, Thaha M, Chan CL. The persistent challenge of parastomal herniation: a review of the literature and future developments. Colorectal Dis. 2013;15(5):e202-214.

19. Krishnamurty DM, Blatnik J, Mutch M. Intestinal stoma: stoma complications. Clin Colon Rectal Surg. 2017;30(3):193.

20. Murken DR, Bleier JIS. Ostomy-related complications. Clin Colon Rectal Surg. 2019;32(3):176-182. 